\title{
An XMM-Newton view of a small sample of Seyfert 1 Galaxies
}

\author{
M. V. Cardaci ${ }^{1,2}$ G. F. Hagele ${ }^{1,2}$, M. Santos-Lleó ${ }^{3}$, Y. Krongold ${ }^{4}$, \\ A. I. Díaz ${ }^{1}$ and P. Rodriguez-Pascual ${ }^{3}$ \\ ${ }^{1}$ Universidad Autónoma de Madrid, Cantoblanco, 28049-Madrid, Spain \\ ${ }^{2}$ Facultad de Cs. Astronómicas y Geofísicas, Universidad Nacional de La Plata, Paseo del \\ Bosque s/n, 1900 La Plata, Argentina \\ ${ }^{3}$ XMM-Newton Science Operation Centre, ESAC, ESA, PO Box 50727, 28080 Madrid, Spain \\ ${ }^{4}$ Instituto de Astronomía, Universidad Nacional Autónoma de México, Apartado Postal \\ 70-264, 04510 México DF, México \\ email: monica.cardaci@uam.es
}

\begin{abstract}
We present a detailed analysis of all the X-ray data taken by the XMM-Newton satellite of a small sample of five Seyfert 1 galaxies: ESO 359-G19, HE 1143-1810, CTS A08.12, Mkn 110, and UGC 11763. Our aim is to characterize the different components of the material that print the absorption and emission features in the X-ray spectra of these objects. The continuum emission was studied through the EPIC spectra taking advantage of the spectral range of these cameras. The high resolution RGS spectra were analyzed in order to characterize the absorbing features and the emission line features that arise in the spectra of these sources.
\end{abstract}

Keywords. galaxies: active, galaxies: Seyfert, galaxies: individual (UGC 11763, ESO 359-G19, HE 1143-1810, CTS A08.12, Mkn 110), X-rays: galaxies

\section{Introduction}

The XMM-Newton satellite is mainly devoted to perform observations in the X-ray band. On-board this satellite are three EPIC cameras that take images and medium resolution spectra, two high resolution spectrographs (RGS), and one optical monitor that can take images or spectra in some optical-UV bands.

A Seyfert 1 is a radio quiet active galactic nucleus (AGN). Their hard X-ray radiation can be characterised with a power law plus some lines produced by iron, for example, the Fe $\mathrm{K} \alpha$ line at $6.4 \mathrm{keV}$. The hard power law describes also the continuum in the soft band only in about the $50 \%$ of the objects. For the rest it is necessary to add another component to account for the extra flux observed. On this soft band one can find as well the transitions involving He-like and H-like atomos of, among other, Oxygen, Neon, and also absorptions of heavier elements that are produced in clouds of partially ionized gas in movement.

The aim of this work is to be able to characterize the X-ray continuum emission of the Seyfert 1 AGNs and to identify possible emission and absorption features in the X-ray spectra and infer the physical conditions of the material in which they are produced. To do this, we select a small sample of Seyfert 1 galaxies and we perform detailed analysis of all data taken during the XMM-Newton observations.

\section{The sample}

We selected a small sample of five objects (Table 1) with a reliable classification as Seyfert 1, that are located at galactic coordinates where the Hydrogen column densities 
Table 1. Journal of observations.

\begin{tabular}{lcccc}
\hline & $\mathrm{nH}\left(10^{20} \mathrm{~cm}^{-2}\right)$ & Obs. date & $\mathrm{z}$ & $\mathrm{T}_{\text {exp }}(\mathrm{s})$ \\
\hline UGC 11763 & 4.67 & 16 May 2003 & 0.063 & 39000 \\
ESO 359-G19 & 1.02 & 09 March 2004 & 0.056 & 24000 \\
HE 1143-1810 & 3.40 & 08 June 2004 & 0.033 & 31000 \\
CTS A08.12 & 4.07 & 30 Oct. 2004 & 0.029 & 46000 \\
Mkn 110 & 1.42 & 15 Nov. 2004 & 0.035 & 47000 \\
\hline
\end{tabular}

are low (about $10^{20} \mathrm{~cm}^{-2}$, which is important for having mainly unabsorbed soft X-rays information), and that are also bright X-rays objects (as seen by other missions). This kind of objects are variable in scale times of days. We pick two objects in a moderate to high historical flux state (HE 1143-1810 and Mkn 110) and the other three in a quite low historical flux state.

\section{Results and Conclusions}

From the detailed analysis of the X-ray data taken by the XMM-Newton satellite for these five objects we find that the parameters that define all the continuum components take values that are well within the ranges found in other Seyfert 1 and QSO (Piconcelli et al. 2005). The indexes of the hard power laws range beteewen 1.2 and 1.8; the lowest values corresponding to the two brightest objects. The soft excess is modelled with a black body component for the lower flux objects with temperatures well within the ranges found in other Seyfert 1 and QSO (kT 80 - 130 keV).

The Fe-K $\alpha$ line was detected in all the five objects, and it is in general weak. A few emission lines were also detected in the soft X-ray band, mainly Oxygen lines such as the OVII He $\alpha$ triplet, detected on four of the objects (even though not all the components are always found). Only in UGC 11763 Ne IX and Fe XVIII lines have been found. In fact UGC 11763 is the only one that shows significant spectral structure, with features both in absorption and emission. Two more objects (CTS A08.12 and Mkn 110) show hints of weak absorption, although not significant.

The absorbing material is mainly detected thanks to the presence of a broad absorption feature around $15-17 \AA$ (see Figure 1), attributed to an unresolved transition array (UTA) resulting from a blend of lines from iron L. The photoinization code used to model the absorption components was developed by Krongold et al., (2003) and the main parameters are: the ionization parameter $(\mathrm{U})$, the column density of the absorbing media and the velocity of the material.

Analysing the absorption features in the UGC11763 spectra we find two partially ionized absorbing components, with distinct ionization states (log $U \sim 1.65$ and 2.6), and with the same velocity respect to the nucleus, (Cardaci et al., 2009).

Placing the absorbing components on the thermal equilibrium curve (see Figure 1), we find that both components lay on stable parts of the curve which is consistent with having the same gas pressure. Adding also the fact that they are kinematically indistinguishable, we conclude that they could constitute two phases of the same medium.

This is a very interesting result because the temperature of the low ionization component $\left(\mathrm{T} \sim 1.8 \times 10^{5} \mathrm{~K}\right)$ is an order of magnitude higher than those found in other Seyfert galaxies meaning that the ions producing the UTA feature are different: in our case Fe XIII to Fe XV and in the other galaxies Fe VII to Fe XII. We have to note that only gas at such high temperature could coexist in pressure equilibrium with the High Ionization Component. 


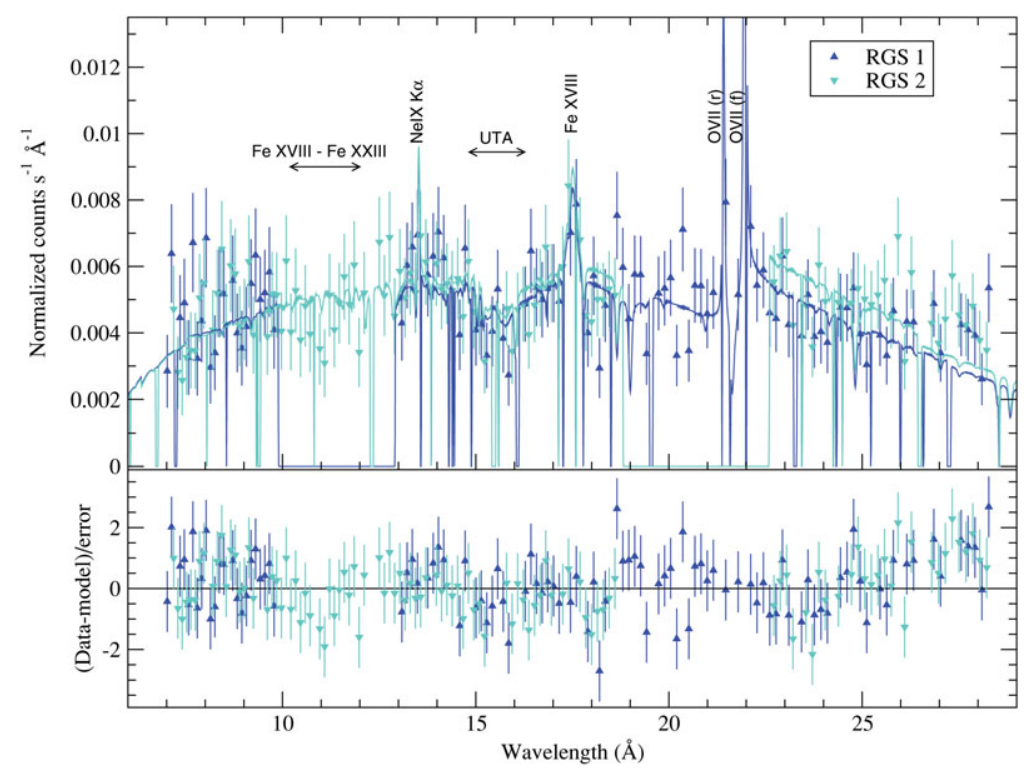

Figure 1. High resolution X-ray spectra -in the rest frame- of UGC 11763 as obtained with RGS1, (blue up triangles), and RGS2 (cyan down triangles), and binned to 15 channels per bin. Solid lines are the convolution of the best fit model with instrument responses.

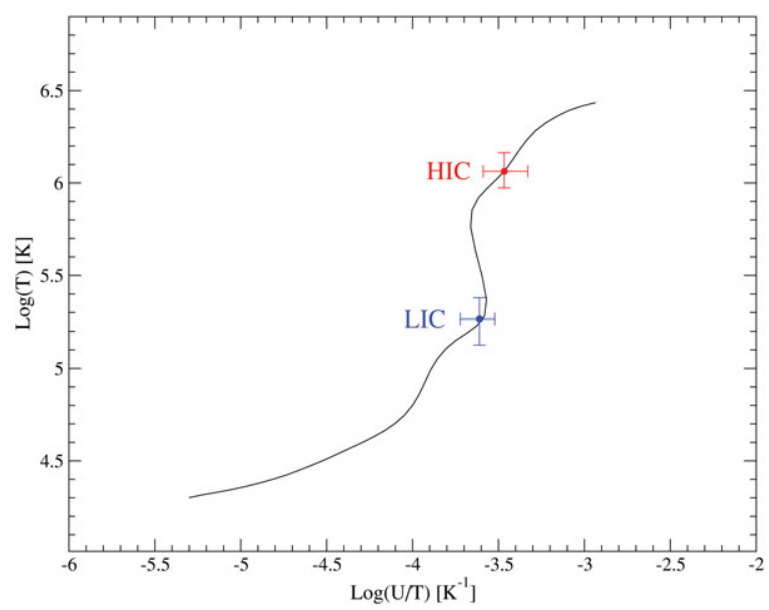

Figure 2. Thermal stability curve of UGC 11763 obtained using the SED based on the simultaneous multiwavelength data obtained in this observation and assuming a column density of $\log N_{\mathrm{H}}=21.5 \mathrm{~cm}^{-1}$. We have indicated the position of the two components of photoionized absorbing material.

\section{Summary}

Regarding the absorbing material around the central engine of the AGNs studied, we find that only one out of five objects shows clear signs of partially ionized (warm) material.

We could characterize the physcial conditions of material around UGC 11763. For which we find two absorbing components that are consistent with their being in pressure equilibrium, i.e., two phases of the same media (UTA of higher ionization than those found in other AGNs). 


\section{References}

Cardaci, M. V., Santos-Lleó, M., Krongold, Y., Hägele, G. F., Díaz, A. I., \& Rodríguez-Pascual, P. 2009, A\&A 432,15

Krongold, Y., Nicastro, F., Brickhouse, N. S., Elvis, M., Liedahl, D. A. \& Mathur, S. 2003, ApJ, 597,832

Piconcelli, E., Jimenez-Bailón, E., Guainazzi, M., Schartel, N., Rodríguez-Pascual, P. M. \& Santos-Lleó, M. 2005, A\&̇A, 432, 15 